\title{
Correction to: Clinical and radiological hip parameters do not precede, but develop simultaneously with cam morphology: a 5-year follow-up study
}

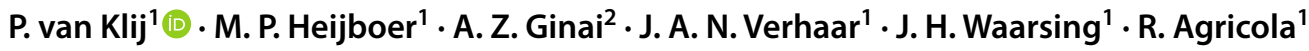

Published online: 5 February 2021

(c) European Society of Sports Traumatology, Knee Surgery, Arthroscopy (ESSKA) 2021

\section{Correction to: \\ Knee Surgery, Sports Traumatology, Arthroscopy https://doi.org/10.1007/s00167-020-06282-0}

Due to typographic mistakes made by the editorial office, several corrections have been made.

The original article has been corrected.

\begin{abstract}
Methods Young male football players participated at baseline $(n=89,12-19$ years of age), 2.5-year $(n=63)$ and 5 -year follow-up $(n=49)$. StandardiZed anteroposterior pelvic and frog-leg lateral radiographs were obtained at each time-point. Cam morphology was quantified BY an alpha angle $\geq 60^{\circ}$, and large cam morphology $\geq 78^{\circ}$.
\end{abstract}

Results A lower NSA, a higher EE and limited IR were consistently associated with cam morphology at all three time-points. These differences were more pronounced in hips with large cam morphology. No association between cam morphology and the LCEA was found. None of the parameters studied preceded cam morphology development.

Conclusion Cam morphology developed simultaneously with a varus orientation, growth plate extension towards the femoral neck and limited hip internal rotation.

The original article can be found online at https://doi.org/10.1007/ s00167-020-06282-0.

P. van Klij

p.vanklij@erasmusmc.nl

1 Department of Orthopaedic Surgery, Erasmus MC, University Medical Center Rotterdam, PO Box 2040, 3000 CA Rotterdam, The Netherlands

2 Department of Radiology, Erasmus Medical Center Rotterdam, Rotterdam, The Netherlands

\section{Introduction}

Cam morphology development also depends on growth plate orientation, when the growth plate extends toward the neck. This results in a stimulus for bone formation at the ante-rolateral head-neck junction. Not only the orientation of the growth plate, but also varus/valgus orientation might influence the stress distribution through the growing proximal femur and thereby the risk of cam morphology development [6, 25].

\section{Materials and methods}

The Medical Ethical Committee of the Erasmus Medical Center (Rotterdam, The Netherlands) approved this study (IRB: NL28614.078.09). Written consent was obtained from all participants. For participants aged under 18 years, written consent from at least one parent was also obtained. The inclusion and exclusion criteria for this study have been described previously $[1,3]$. Adolescent male football players who played in selection teams of Feyenoord football club in Rotterdam (The Netherlands) were included. Exclusion criteria were any known hip disorder. At baseline, information letters were sent to all eligible asymptomatic athletes $(n$ =141), of whom 101 gave informed consent and 89 (12-19 years of age) joined this study at baseline. At 2.5-year followup, 63 participants were included and at 5-year follow-up, 49 participants (mean age, $20.5 \pm 2.2$ years) (Fig. 1). The 5-year follow-up was performed between June and October 2015.

\section{Radiographs}

A standardiZed radiographic protocol was used at baseline, 2.5- and 5-year follow-up, which has been described previously [1, 3]. In summary, three radiographs of the hip were obtained: a standardiZed supine anteroposterior (AP) radiograph of the pelvis and a frog-leg lateral radiograph of each hip. 


\section{Radiographic and clinical parameters}

While maintained in neutral rotation, the first resistance/end feel during passive internal rotation was measured in supine position on a flat examination table with a gonio-meter.

\section{Statistical analysis}

The NSA, EE, LCEA and internal rotation were studied in a longitudinal design to observe if there were any differences in these values at baseline between football players that did or did not develop cam morphology, using a GEE model with logistic links function, adjusted for age and BMI. The unadjusted data are presented in a sensitivity analysis (Supplemental Table 1). SPSS25.0 (Windows) was used for statistical evaluation.

Table 1 Demographic data and cam morphology prevalence at baseline, 2.5-year follow-up and 5-year follow-up

\begin{tabular}{|c|c|c|c|}
\hline $\begin{array}{l}\text { Participant charac- } \\
\text { teristics }\end{array}$ & Baseline $(n=89)$ & $\begin{array}{l}2.5 \text {-year } \\
\text { follow-up ( } n \\
=63)\end{array}$ & $\begin{array}{l}\text { 5-year } \\
\text { follow-up }(n \\
=49)\end{array}$ \\
\hline Age, year & $15.2 \pm 2.0$ & $17.3 \pm 2.0$ & $20.5 \pm 2.2$ \\
\hline Weight, kg & $59.4 \pm 13.8^{\wedge}$ & $68.4 \pm 11.1^{\#}$ & $73.8 \pm 7.9$ \\
\hline Height, $\mathrm{cm}$ & $170.3 \pm 12.2^{\wedge}$ & $177.44 \pm 8.0^{\#}$ & $180.3 \pm 6.6$ \\
\hline $\begin{array}{l}\text { Body mass index } \\
\mathrm{kg} / \mathrm{m}^{2}\end{array}$ & $20.1 \pm 2.3^{\wedge}$ & $21.6 \pm 2.2^{\#}$ & $22.7 \pm 1.6$ \\
\hline $\begin{array}{l}\text { Football experi- } \\
\text { ence, year }\end{array}$ & $9.0 \pm 2.54^{\wedge}$ & $11.1 \pm 2.54^{\#}$ & $14.3 \pm 2.68$ \\
\hline $\begin{array}{l}\text { Training intensity, } \\
\text { h/week }\end{array}$ & $8.0 \pm 1.8^{\wedge}$ & $8.7 \pm 1.9^{\# \#}$ & $9.3 \pm 2.9$ \\
\hline $\begin{array}{l}\text { Cam morphology } \\
\text { prevalence per } \\
\text { hip }\end{array}$ & 178 hips & 126 hips & 98 hips \\
\hline Cam & $87(48.9 \%)$ & $86(68.3 \%)$ & $78(79.6 \%)$ \\
\hline Large cam & $24(13.5 \%)$ & $25(19.8 \%)$ & $25(25.5 \%)$ \\
\hline
\end{tabular}

Due to missing data, data of $n=87\left({ }^{\wedge}\right), n=58$ (\#) and $n=57$ (\#\#) are presented

Values are expressed as mean \pm standard deviation, with $n=$ participants

\section{Discussion}

This study found that increasing varus orientation of the hip was associated with cam morphology presence and size.

In this study, an increased EE was cross-sectionally associated with cam morphology, but did not precede cam morphol-ogy development. This is in line with Siebenrock et al. [29] who described an association between epiphyseal extension and cam morphology in a group of 15 participants with cam morphology compared with 15 controls. Three other studies also found a correlation between epiphyseal extension and the alpha angle $[14,22,27]$. There is no previous longitu-dinal study available on EE and the development of cam morphology DEVELOPMENT. These clinical studies fit with a finite element study [25], which showed higher shear stresses (a trigger for bone formation) at the location where cam morphology develops when the growth plate extended towards the femoral neck.

The differences in internal rotation observed between hips with and without cam morphology range from 3 to 6 degrees, depending on cam morphology size.

Publisher's Note Springer Nature remains neutral with regard to jurisdictional claims in published maps and institutional affiliations. 